\title{
Valorisation Agricole D’un Compost Produit À Partir Du Compostage En Cuve Des Déchets Municipaux
}

\section{Chennaoui}

Centre régional des métiers de l'éducation et de la formation (CRMEF), Laboratoire des Sciences de la Vie et de la Terre (SVT), El Jadida, Maroc Laboratoire de l'Eau et Environnement, Faculté des Sciences, Université Chouaïb Doukkali, El Jadida, Maroc

\section{Y. Salama}

A. Makan

Consulting, Environnement, Qualité et Sécurité, 33 Hay Amal, Khouribga, Maroc

\section{Mountadar}

Laboratoire de l'Eau et Environnement, Faculté des Sciences, Université Chouaïb Doukkali, El Jadida, Maroc.

doi: 10.19044/esj.2016.v12n35p247 URL:http://dx.doi.org/10.19044/esj.2016.v12n35p247

\begin{abstract}
This work is in the context of the management and recycling of solid urban waste. The evolution of the physicochemical and microbiological parameters during composting pilot scale household waste was studied. The maturity of the processes and the agronomic value of the finished product were examined.

The results showed that the organic fraction of solid waste could be composted successfully in 60 days and revealing a vigorous microbial activity. The final compost has been satisfactory for its agricultural application. Phytotoxicity tests conducted on wheat and tomato crops, show that the incorporation of $25 \%$ culture support the compost allows a germination rate of up to $85 \%$ against $70 \%$ for the control for the variety of corn. In the case of tomatoes, we recorded a germination rate of about $67 \%$ against $58 \%$ for the control.
\end{abstract}

Keywords: Waste, compost, recycling, maturity test 


\section{Résumé}

Le présent travail s'inscrit dans le contexte de la gestion et la valorisation des déchets solides urbains. L'évolution des paramètres physicochimiques et microbiologiques au cours du compostage à l'échelle pilote des déchets ménagers a été étudiée. La maturité du process et la valeur agronomique du produit fini ont été examinées.

Les résultats obtenus ont montré que la fraction organique des déchets solides pourrait être composté avec succès dans un délais de 60 jours et révélant une activité microbienne vigoureuse. Le compost final a été satisfaisant pour son application agricole. Les essais de phytotoxicité menés sur des cultures de blé et de la tomate, révèlent que l'incorporation de $25 \%$ du compost au supports de culture permet un taux de germination allant jusqu'à $85 \%$ contre $70 \%$ pour le témoin pour la variété du blé. Dans le cas de la tomate, nous avons enregistré un taux de germination de l'ordre de $67 \%$ contre $58 \%$ pour le témoin.

Mots clés : Déchets, compost, valorisation, test de maturité

\section{Introduction}

Le Maroc doit faire face aujourd'hui à une augmentation de la quantité de ses déchets qui ne cesse de s’accentuer. En effet, la génération totale de déchets solides est de 6,852 million de tonnes métriques (SWEEP NET, 2014). La génération de déchets urbains est actuellement environ 0,67 kilos par jour par habitant. La collecte des déchets solides urbains est régulière et presque quotidienne, elle est estimée à 5,5 million de $\mathrm{T}$ par an. En 2013, la collecte des déchets solides urbains couvre à peu près les $74 \%$ des déchets générés dans des superficies urbaines. Or, les ordures ménagères se caractérisent par la prédominance des déchets fermentescibles (déchets végétaux et de cuisine) et par leur humidité élevée (Djerrari, 1993 ; El M'ssari, 1993 ; Soudi et al., 1995) et leur recyclage par compostage, est actuellement considéré comme une des composantes du développement durable (Basalo, 1974 ; Golueke, 1977 ; Golueke, 1979).

L'introduction du compost mûr dans le sol est une solution pour le maintien de la matière organique dans le sol (Tiejen, 1975). Le compost est, en effet, un produit riche en matières organiques et en composés minéraux, capable d'améliorer la fertilité du sol.

L'objectif de ce travail consiste à étudier l'évolution des propriétés chimiques, physiques et microbiologiques des déchets ménagères au cours du compostage en cuve et la valorisation agronomique du produit fini. 


\section{Matériels et méthodes}

\section{Origine et caractérisation des déchets}

Sur la base de la consommation marocaine, les déchets organiques ou déchets verts à composter seront constitués de 37\% déchets de légumes, 35\% des déchets de fruits, $13 \%$ des produits de thé de déchets et $15 \%$ des autres déchets.

Un échantillon de $20 \mathrm{~kg}$ a été pesé et manuellement haché en petits morceaux de 2 à $5 \mathrm{~mm}$ de longueur. Les déchets obtenus, homogénéisés avec $1 \mathrm{~kg}$ de terreau pour l'apport de la microflore, ont été transférés dans le bioréacteur. L'ajustement de l'humidité a été réalisée soit en ajoutant la quantité requise de l'eau ou par le séchage du mélange, sous les rayons du soleil, s'il y a un excès d'humidité.

\section{Description du bioréacteur}

Les figure 1 et 2 montrent le bioréacteur en laboratoire qui a été spécialement conçu et utilisé pour le compostage des déchets dans cette étude. Le bioréacteur est un fut métallique vertical d'un volume d'environ 200 litres. L'épaisseur du métal est de $1 \mathrm{~mm}$. Le bioréacteur est conçu avec une ouverture dans la face supérieure pour l'introduction des déchets. Le bioréacteur est munit d'une soupape pour injecter et une pour retirer l'air et un robinet pour le lexiviat. L'étanchéité est assurée par un joint en caoutchouc.

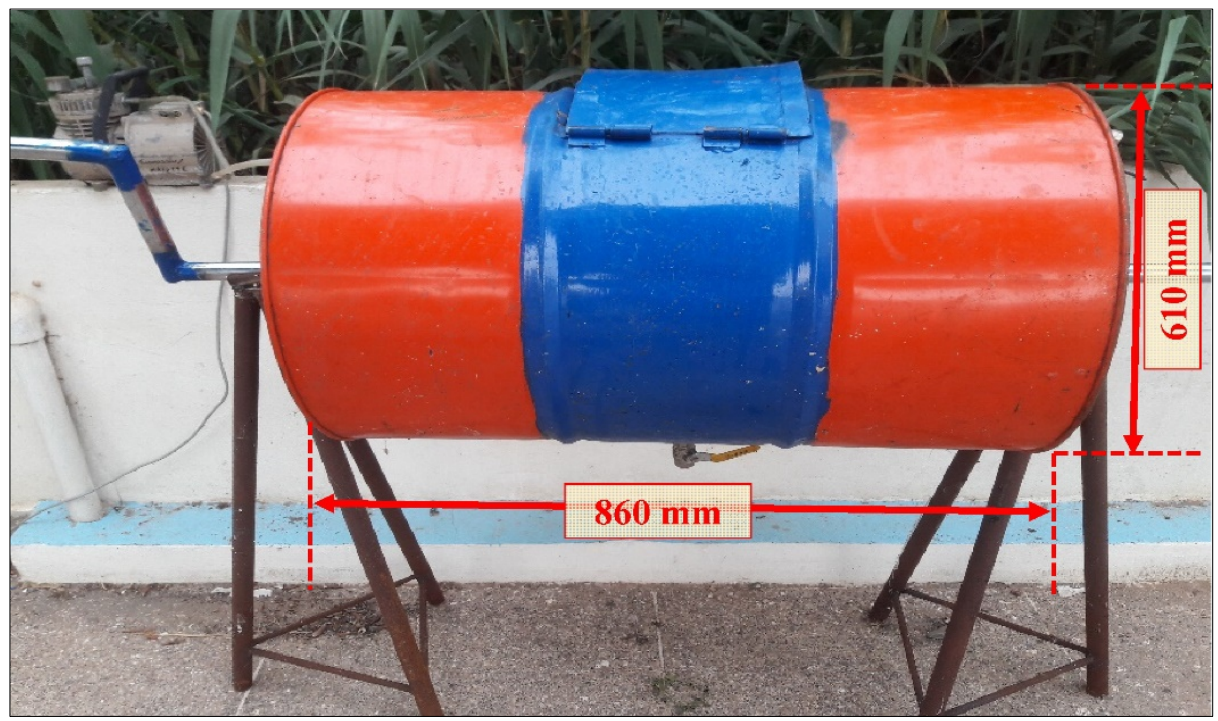

Figure 1 : Bioréacteur pilote utilisé pour l'expérimentation 


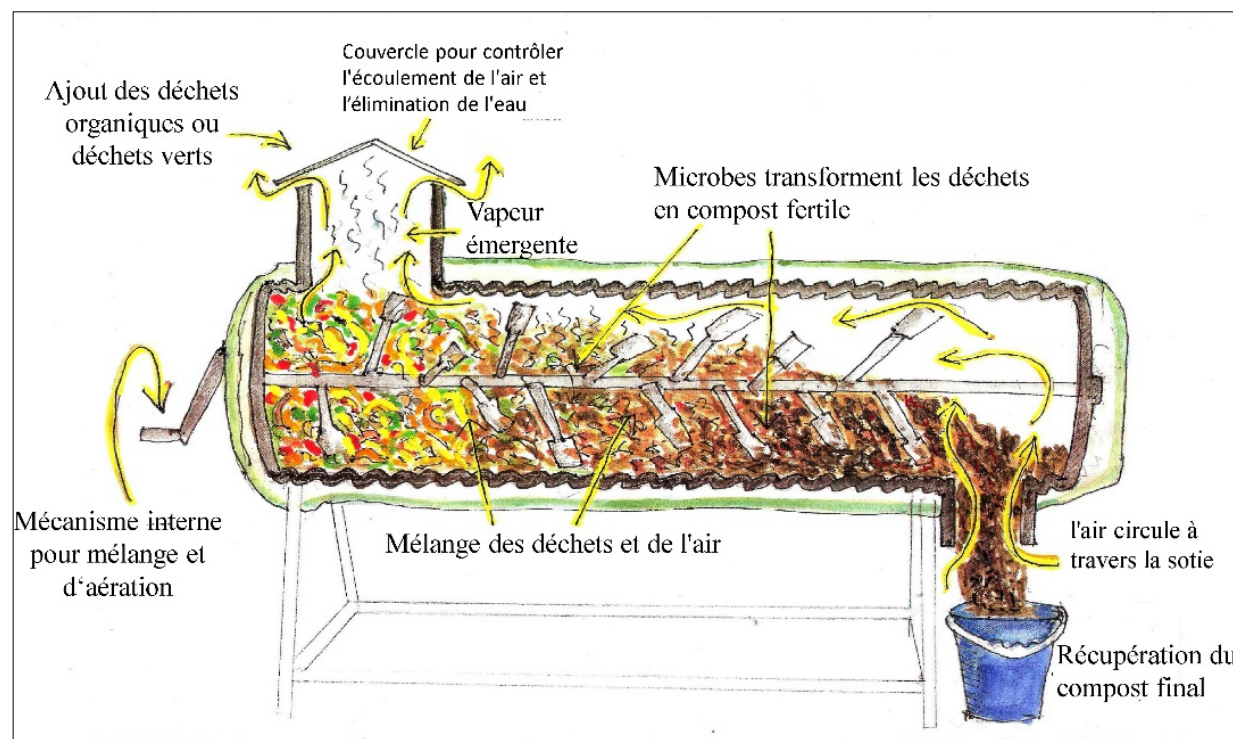

Figure 2 : Schéma descriptif du fonctionnement du Bioréacteur

\section{Conditions de compostage}

Le bioréacteur a été alimenté d'air tous les jours et les gaz formés au cours du processus de la dégradation ont été évacués à la fin de la journée. Ce processus est répété jusqu'à la stabilisation du compost. Le bioréacteur est ensuite retourné de manière périodique afin d'assurer une bonne aération du tas et permettre une fermentation aérobique. Le processus de compostage a pris environ 60 jours jusqu'à la stabilisation. Enfin, les valeurs des indicateurs nécessaires du processus ont été mesurés.

\section{Préparation des échantillons pour analyses}

Les échantillons sont prélevés à l'occasion du retournement. Le prélèvement de $100 \mathrm{~g}$ de matière dans des sacs stériles, est réalisé 3 fois à différents niveaux du tas de manière à obtenir un échantillon aussi représentatif que possible.

\section{Les méthodes d'analyse des paramètres physico-chimiques \\ Température}

La température a été mesurée en utilisant un thermomètre numérique Multi-Système (ST-9283B; Indiamart, Delhi, Inde).

\section{- $\quad$ Humidité}

La teneur en humidité a été mesurée après séchage de l'échantillon à $105^{\circ} \mathrm{C}$ pendant une nuit.

\section{- Matière organique}

La matière organique (MO) a été calculé à partir des cendres après séchage d'un échantillon de $20 \mathrm{~g}$ de poids sec à $550^{\circ} \mathrm{C}$ pendant $6 \mathrm{~h}$. 
- $\quad$ Carbone organique total et azote total kjeldahl

Carbone organique total (COT) et azote total Kjeldahl (NTK) ont été mesurés, respectivement par la méthode Walkley-Black et la méthode Kjeldahl. Le rapport $\mathrm{C} / \mathrm{N}$ est alors calculé en fonction de la concentration en COT et NKT.

\section{- $\quad$ pH et conductivité}

Le $\mathrm{pH}$ et la conductivité électrique (CE) ont été mesurées par mélange échantillon-eau (poids : volume $=1: 10$ ). Les valeurs ont été lues respectivement sur le pH-522 WTW mètre (marque Xylem, Weilheim, Allemagne) et sur EC-214 conductimètre (HANNA Instruments, Agadir, Maroc).

\section{- $\quad$ Ammonium et nitrate}

Ammonium $\mathrm{NH}_{4}^{+}$d'azote a été mesurée par spectrophotométrie d'acide salicylique et de l'hypochlorite de sodium (Lu, 2000). Nitrate $\mathrm{NO}_{3}^{-}$a été déterminée en utilisant la chromatographie ionique (Wei, 2002).

Toutes les analyses ont été triplées afin d'assurer la reproductibilité et la représentativité de l'échantillon.

\section{Les méthodes d'analyse des paramètres microbiologiques}

Dans un Erlenmeyer de $250 \mathrm{ml}$ contenant $90 \mathrm{ml}$ d'eau distillée stérile sont ajoutés aseptiquement $10 \mathrm{~g}$ de compost sec (après séchage à $30^{\circ} \mathrm{C}$ pendant une nuit). Ce mélange est agité mécaniquement à l'aide de barreaux magnétiques pendant 30 minutes le but de libérer le maximum de la charge microbienne. La suspension obtenue correspond à la dilution $10^{-1} .10 \mathrm{ml}$ de la dilution $10^{-1}$ sont prélevés aseptiquement et mis dans $90 \mathrm{ml}$ d'eau distillée stérile donnant ainsi la dilution $10^{-2}$ qui est agitée pendant deux minutes avant de prélever $10 \mathrm{ml}$ que l'on ajoute à $90 \mathrm{ml}$ d'eau distillée stérile et ainsi de suite jusqu'à la dilution $10^{-8} .1 \mathrm{ml}$ est prélevé à partir de chaque dilution, en opérant de la dilution $10^{-8}$ à la dilution $10^{-1}$, et ensemencé sur les différents milieux de culture, à l'aide d'un étaloir de verre stérile.

\section{- Microflore bactérienne totale}

Le dénombrement de la flore mésophile aérobie totale (FMAT) et de la flore thermophile (FT) est fait par comptage des colonies sur le milieu Plate Count Agar et incubés respectivement à $35^{\circ} \mathrm{C}$ et $55^{\circ} \mathrm{C}$.

\section{Microflore fongique}

L’analyse a été conduite selon la technique des suspensions-dilutions telle qu'elle est décrite par (Rapilly, 1968). La culture est faite sur le milieu solide spécifique PDA additionné d'un antibiotique le chloramphénicol (Sigma) à raison de $5 \mu \mathrm{g} / \mathrm{ml}$. Les boîtes sont incubées à $30^{\circ} \mathrm{C}$ à l'obscurité pendant 3 jours au bout desquels le comptage des colonies est réalisé, puis les boîtes sont placées sous lumière blanche continue afin de favoriser la pigmentation des colonies tout en notant l'apparition de nouvelles colonies. 
Expression des résultats : La détermination de la charge fongique est faite par comptage des colonies et les résultats sont exprimés en UFC (nombre d'Unités Formant Colonies) / g de compost selon la formule mathématique ci-dessous. Seules les boîtes contenant entre 15 et 30 colonies au niveau de deux dilutions successives sont retenues pour le dénombrement (Dutruc-Rosset, 2003).

$$
\mathrm{N}=\frac{\Sigma \text { colonies }}{V X(n 1+0,1 n 2) X d 1}
$$

Où : N: Nombre d'UFC par gramme de compost;

$\Sigma$ colonies: Somme des colonies des boîtes interprétables;

V: Volume de solution déposée (1ml);

n1 : Nombre de boîtes considéré à la première dilution retenue;

n2: Nombre de boîtes considéré à la seconde dilution retenue;

d1 :Facteur de la première dilution retenue.

\section{Tests de phytotoxicité}

Dans cette étude, deux tests de phytotoxicité ont été utilisés. Le test de germination et le test d'indice de germination sur différentes cultures (blé, tomate, laitue) avec le compost produit.

Test de germination (Organisation Mondiale de la Santé, 1978)

Ce test est basé sur le pouvoir germinatif des graines de deux plantes : blé et tomate. Il consiste à semer un même nombre (10) de graines dans des pots contenant différents pourcentages de composts et de sables. Le taux de germination est évalué par rapport au témoin (100\% sable). Les différentes proportions des composts et sables sont : $100 \% \mathrm{~S}, 75 \% \mathrm{~S}+25 \% \mathrm{C}$, $50 \% \mathrm{~S}+50 \% \mathrm{C}, 25 \% \mathrm{~S}+75 \% \mathrm{C}, 100 \% \mathrm{C}$ (S: sable et C: compost). Les différentes conditions de germination des graines de blé et de tomate sont répertoriées dans le Tableau 1.

Tableau 1 : Conditions de germination des graines (blé et tomate).

\begin{tabular}{|c|c|c|c|c|}
\hline Graines & $\begin{array}{c}\mathrm{T}\left({ }^{\circ} \mathrm{C}\right) \text { possible } \\
\text { de germination }\end{array}$ & $\begin{array}{c}\mathrm{T}\left({ }^{\circ} \mathrm{C}\right) \text { Idéale de } \\
\text { germination }\end{array}$ & $\begin{array}{c}\text { Trempage dans } \\
\text { l'eau (Heure) }\end{array}$ & $\begin{array}{c}\text { Germination } \\
\text { (jour) }\end{array}$ \\
\hline Blé & 20 & 20 & 12 & $2-3$ \\
\hline Tomate & 21 & 21 & $12-20$ & $7-8$ \\
\hline
\end{tabular}

Test d'indice de germination (IG) (Zacconi et al.1981)

Le principe consiste à placer des graines de laitue dans une série de boites de Pétri avec du papier filtre imbibé de doses croissantes d'extrait des composts parallèlement à une série témoin (sans extrait de compost). L'ensemble est placé dans une chambre de germination (incubateur) à $27^{\circ} \mathrm{C}$ pendant 24 heures. En fin de la germination, les graines sont comptées et les longueurs des racines sont mesurées. L'indice de germination (IG) est calculé par l'équation suivante : 
$\mathrm{IG}=(\mathrm{GB} / \mathrm{GT}) \times(\mathrm{LB} / \mathrm{LT}) \times 100$

Avec,

IG : Indice de Germination,

GB : Nombre de graines germées dans le cas d'apport du compost,

GT : Nombre de graines germées dans le cas du traitement témoin,

LB : Longueur des racines dans le cas des apports du compost,

LT : Longueur des racines dans le cas du traitement témoin.

Les différentes doses d'extraits de compost et d'eau distillée utilisées au cours de cette expérience sont les suivantes : 100\% E ; 75\% E+ 25\% EC ; $50 \%$ E+ 50\% EC ; 25\% E+ 75\% EC ; 100\% EC (E : Eau ; EC : extrait de compost).

\section{Résultats et discussion}

\section{Evolution de la Température}

La surveillance de la température permet une mesure indirecte de l'intensité de la dégradation aérobie. À des températures inférieures à $20^{\circ} \mathrm{C}$, les micro-organismes psychrotrophes seulement sont actifs. Entre 20 et $40^{\circ} \mathrm{C}$, il est le tour de ceux mésophiles, mais les micro-organismes thermophiles sont actifs seulement à des températures comprises entre 40 et $70^{\circ} \mathrm{C}$ (Mustin, 1987).

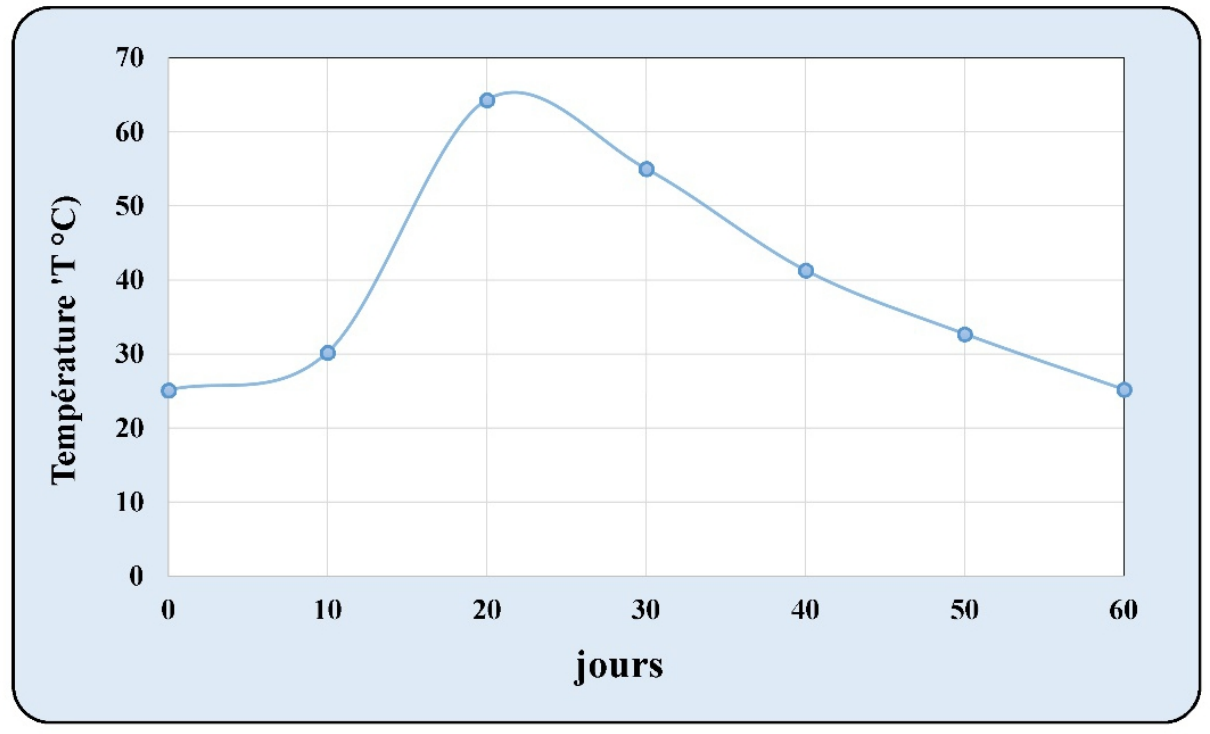

Figure 3 : Evolution de la température durant la période du compostage.

Au début du compostage l'activité biologique s'installe à travers des micro-organismes mésophiles ce qui augmente la température de $25,1^{\circ} \mathrm{C}$ à 30,2 durant le 10e jour (Fig. 3). Cette élévation de la température est la conséquence directe de l'oxydation de la matière organique des substrats (Hassen et al., 2001 ; Ahn, 2009). Cette oxydation permet ainsi la libération 
de l'énergie contenue dans les liaisons chimiques des molécules constitutives (Ryckeboer, 2003).

Avec le compostage, l'intense activité des micro-organismes génère une élévation de la température à $64,3^{\circ} \mathrm{C}$ qui est atteinte au 20e jour et dure environ 6 jours ce qui permet l'installation des microorganismes thermophiles et thermo-tolérants (Chang 1967 ; Chang et al., 1967 ; Strom 1985 ; Beffa et al, 1996).

Cependant, durant la phase de maturation qui succède à la phase thermophile, les pertes de chaleur par échange avec le milieu extérieur et la diminution des éléments nutritifs, engendrent une chute de température progressive et se rapproche de l'ambiante $25,2^{\circ} \mathrm{C}$ après 60 jours de compostage.

\section{Evolution du pH}

Le $\mathrm{pH}$ est un facteur important qui influence la plupart des réactions biochimiques catalysées par des enzymes ce qui permet la biodisponibilité des nutriments et la solubilité des éléments minéraux pour les microorganismes.

Le processus de compostage se caractérise par un $\mathrm{pH}$ initial de 7,5. L’évolution du $\mathrm{pH}$ en fonction du temps montre trois phases différentes (Figure 4). Le compostage passe par une phase acidogène de 20 jours, où le $\mathrm{pH}$ est aux alentours de 5,4. Vers le 40e jour, il y a un passage rapide par une phase de neutralité. Vers le 50e jour, il y a passage par une phase d'alcalinisation où le $\mathrm{pH}$ atteint 7,8. Cette phase est le résultat d'une part d'une production ammoniacale à partir de la dégradation des amines protéiques lors du processus d'ammonification et d'autre part d'une libération des bases auparavant intégrées à la matière organique (Kochtizky et al 1969 ; Gray et al 1971; Peters et al 2000). Cette stabilité est aussi la conséquence de la présence d'ions $\mathrm{Ca}^{2+}$ qui augmentent au cours du compostage suite à l'humification et qui jouent un rôle de tampon dans le milieu (Juste, 1980 ; Morel et al., 1984). Le pH final légèrement alcalin 8,1 fait du compost un produit sans risques pour le sol et pour les plantes. La valeur de $\mathrm{pH}$ obtenue dans cette expérimentation concorde avec les travaux de He et al. (1992) et de Hellmann et al. (1997). 


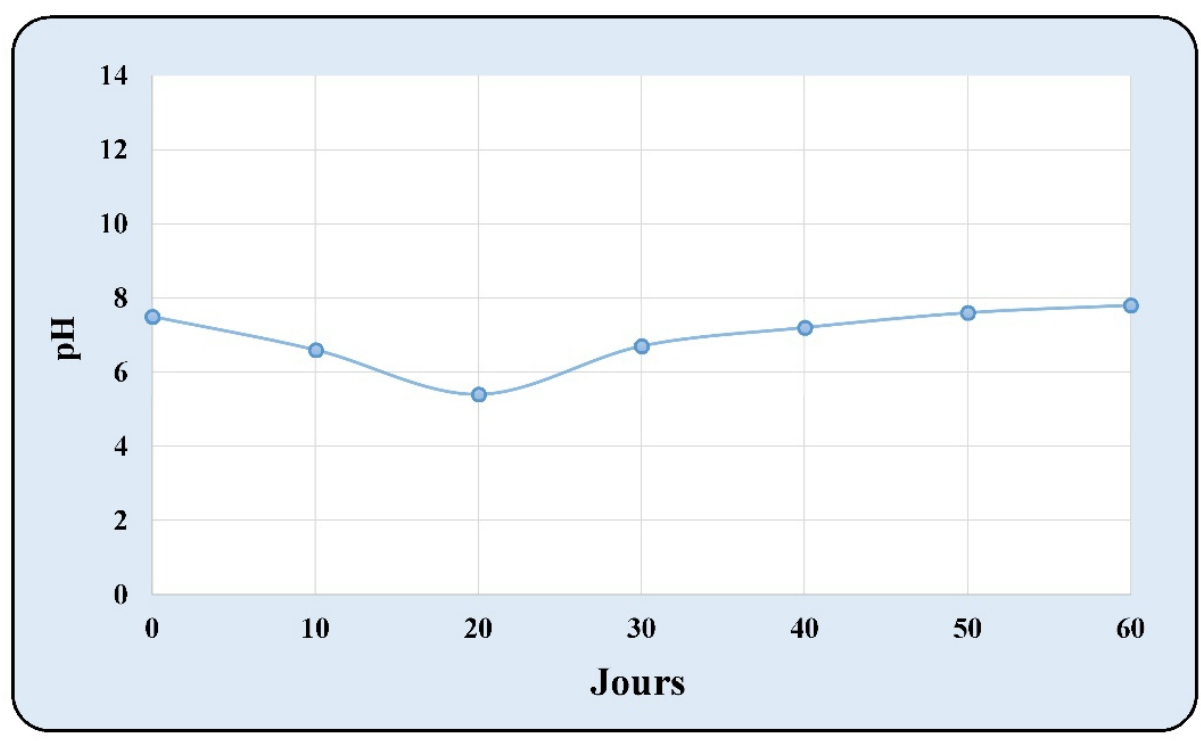

Figure 4 : Evolution du pH durant la période du compostage.

\section{Evolution de l'humidité}

Une bonne humidité est primordiale pour que l'activité des microorganismes soit plus importante, ce qui accélère le processus de compostage. Le taux d'humidité diminue significativement au cours du temps, environ $70 \%$ dans le compost jeune, il n'est plus que de 10\% dans le compost mûr (fig. 5). On attribue cette perte d'eau à la lixiviation et à l'évaporation due à l'élévation de la température due à l'activité microbienne intense lors du compostage (Jemali et al.1996).

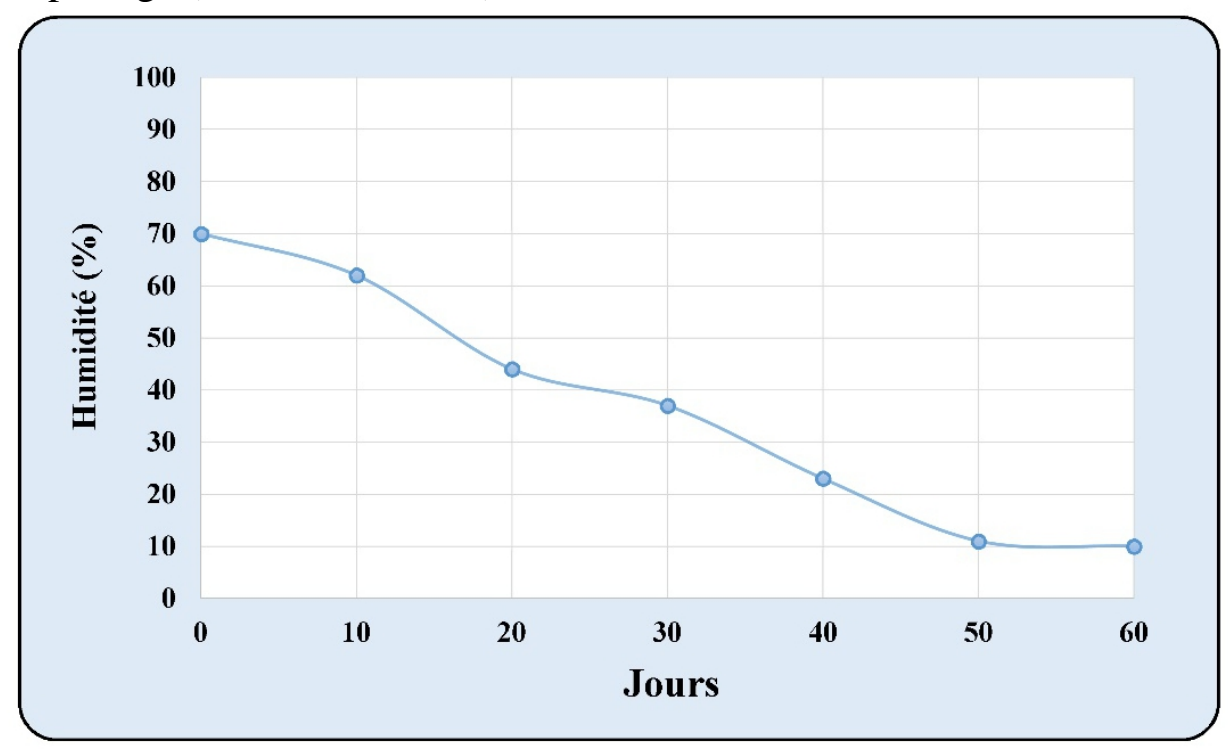

Figure 5 : Evolution de l’humidité durant la période du compostage. 


\section{Evolution de NH4+ et NO3-}

Le contenu $\mathrm{NH}_{4}^{+}$dans la charge d'abord augmente pendant les 20 premiers jours du compostage pour atteindre une valeur de $1363 \mathrm{mg} / \mathrm{kg}$ puis diminue progressivement (Fig. 6), ce qui pourrait expliquer par la décomposition microbienne de la MO contenant de l'azote pour se convertir en ammoniac. La diminution de $\mathrm{NH}_{4}^{+}$est un indicateur d'un bon processus de la maturation. Zucconi et de Bertoldi (1987) ont recommandé une teneur maximale deNH ${ }_{4}^{+}$de $400 \mathrm{mg} / \mathrm{kg}$ en compost mûr. Ainsi, la valeur de $\mathrm{NH}_{4}^{+}$ dans le compost final obtenu répond donc à la demande des applications agricoles.

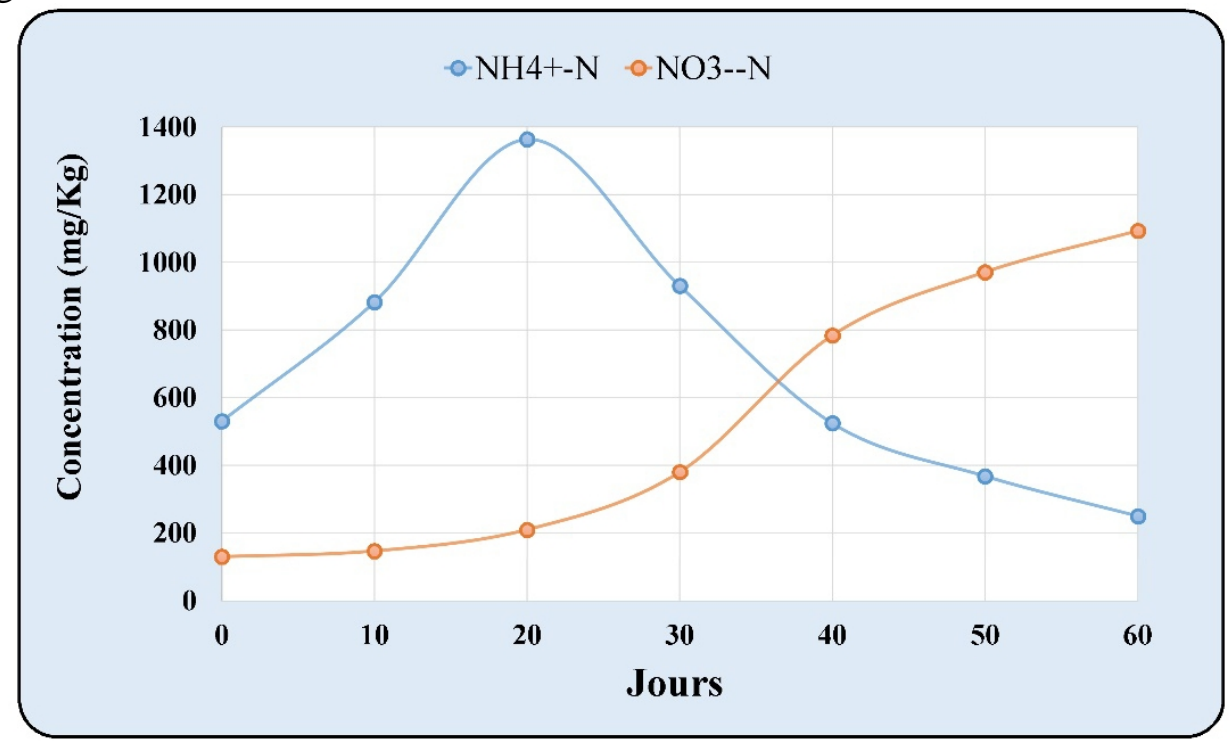

Figure 6 : Evolution de la teneur en $\mathrm{NH}_{4}{ }^{+}$et $\mathrm{NO}_{3}{ }^{-}$durant la période du compostage.

La teneur en $\mathrm{NO}_{3}{ }^{-}$a gardé une tendance de croissance au cours du processus de compostage (fig. 6). Comme la température élevée (plus de 40 ${ }^{\circ} \mathrm{C}$ ) a inhibé l'activité et la croissance des bactéries nitrifiantes dans la phase thermophile, il n'y a aucune augmentation significative en teneur de $\mathrm{NO}_{3}{ }^{-}$ pendant la phase initiale du processus de compostage. Lorsque le processus de durcissement avait commencé après le 20e jour, on a observé une augmentation rapide de la teneur en $\mathrm{NO}_{3}{ }^{-}$. Après cela, le processus de durcissement est terminé le 50e jour et la teneur en $\mathrm{NO}_{3}{ }^{-}$avait tendance à se stabiliser relativement pour atteindre $1093 \mathrm{mg} / \mathrm{kg}$ à la fin du compostage.

\section{Evolution du C/N et de la matière organique MO}

Selon la Fig. 7, le rapport $\mathrm{C} / \mathrm{N}$ présente une déclinaison progressivement à cause de la minéralisation de la matière organique. Le substrat initial à un $\mathrm{C} / \mathrm{N}$ de 27 . Dès que les composés carbonés facilement disponibles ont été épuisés, le taux de réduction $\mathrm{C} / \mathrm{N}$ diminue. Cette 
diminution peut être expliquée par le fait que les micro-organismes consomment plus de carbone (composant principal des molécules organiques) que l'azote. Au début du compostage le rapport $\mathrm{C} / \mathrm{N}$ était d'environ 27 et la fin du processus a été réduit à 11 . Hirai et al., 1983 a proposé un rapport $\mathrm{C} / \mathrm{N}$ est égal ou inférieur à 20 en tant que norme pour le compost mûr. Donc le compost obtenu peut être qualifiés comme un bon compost de qualité qui peut être appliqué dans les terres agricoles. La fraction organique a été minéralisé en composés stables par les activités microbiennes, ce qui explique sa diminution au cours du processus de compostage elle est passé d'environ $92 \%$ à environ $48 \%$ après 60 jours de compostage.

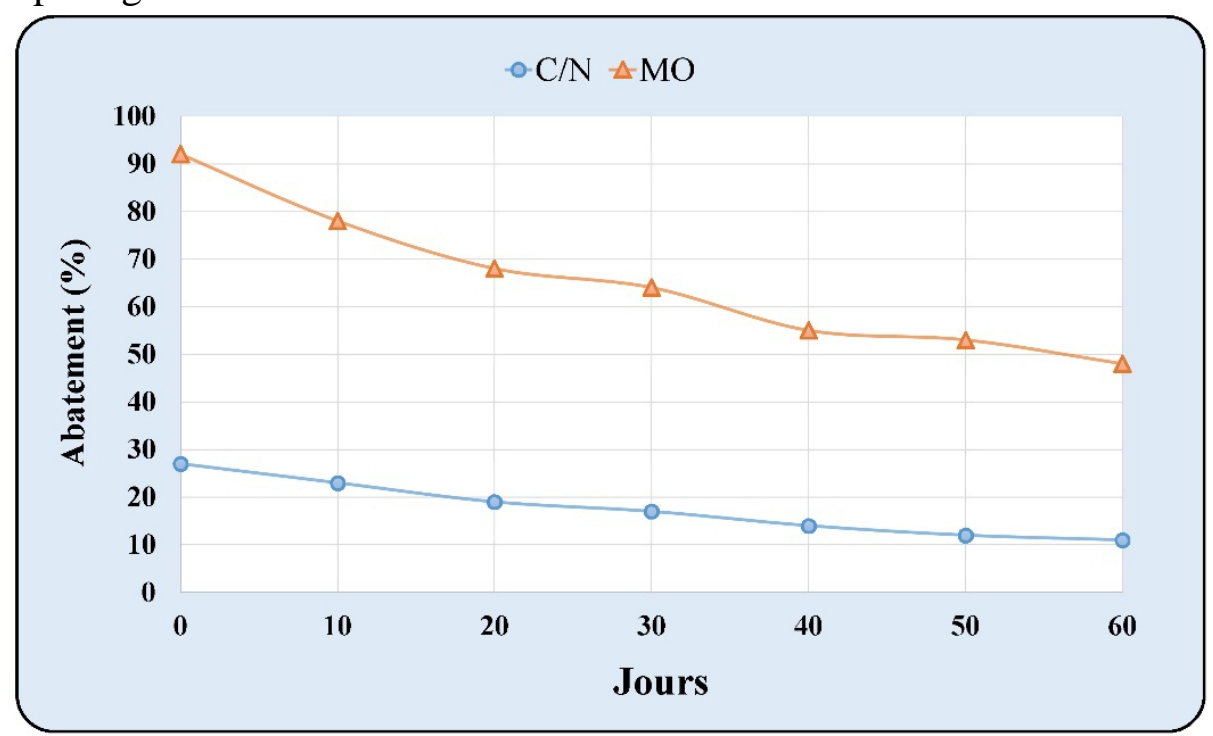

Figure 7 : Evolution du rapport $\mathrm{C} / \mathrm{N}$ et de la teneur en matière organique durant la période du compostage.

\section{Evolution de la CE}

La CE reflète le degré de salinité du compost produit et indique ses possibles effets phytotoxiques/inhibiteurs sur la croissance des plantes utilisé comme engrais (par exemple faible taux de germination, flétrissement, etc...) (Lin 2008). Le compost avec une CE faible peut être utilisé directement alors que le compost de haute CE doit être bien mélangé avec de la terre ou d'autres matériaux à faible $\mathrm{CE}$ avant qu'il puisse être utilisé pour les cultures (Chen 1999). Conformément à la figure 8, la courbe de la teneur en CE représente une augmentation par rapport à la valeur initiale de 4,9 $\mathrm{ms} / \mathrm{cm}$ à un maximum de $7,5 \mathrm{~ms} / \mathrm{cm}$ au 20 e jour, suivie d'une diminution progressive jusqu'à la fin du processus de compostage. L'augmentation initiale de la CE pourrait être causée par la libération de sels minéraux tels que les phosphates et les ions ammonium par la décomposition des 
substances organiques (Gómez-brandon et al., 2008). Pendant la progression du compostage, la volatilisation de l'ammoniac et de la précipitation des sels minéraux peuvent être les raisons possibles de la diminution de la CE jusqu'à la phase finale de compostage (Wong et al., 1995). Le CE du produit de compostage final ne dépasse pas la teneur limite de $3 \mathrm{~ms} / \mathrm{cm}$, ce qui indique que CE ne serait pas nuire à la croissance des plantes (Soumaré et al. 2002).

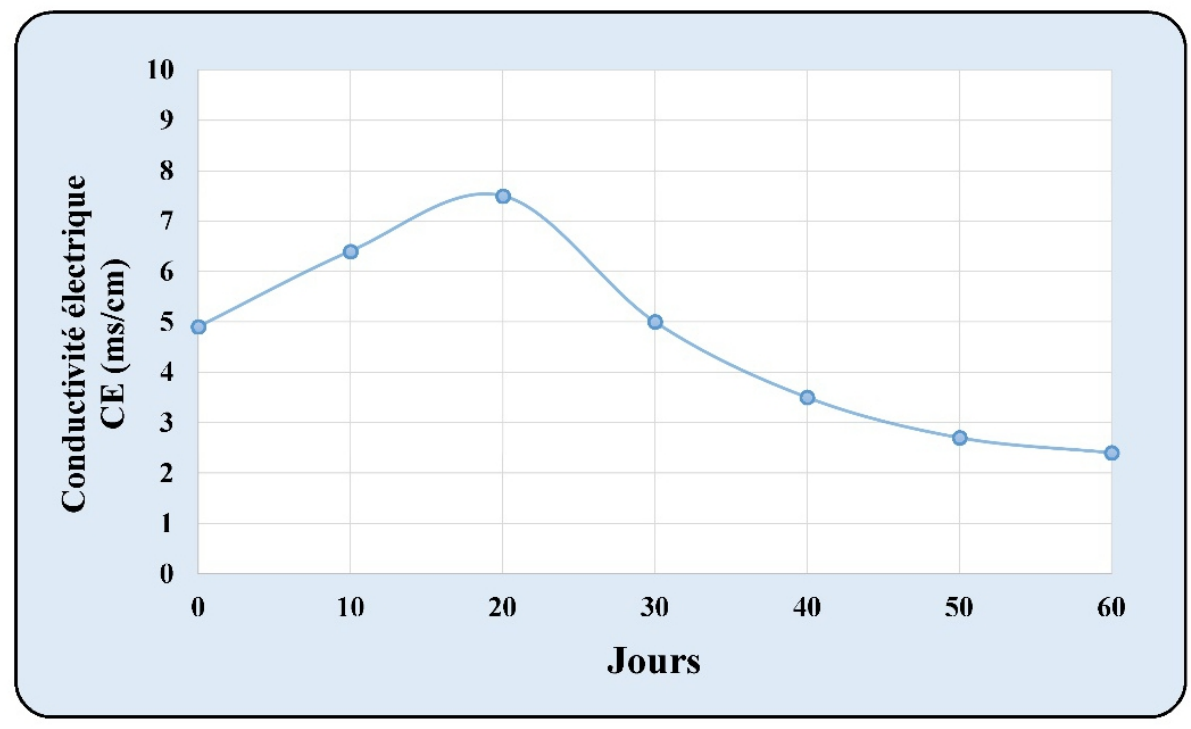

Figure 8 : Evolution de la conductivité électrique durant la période du compostage.

\section{Evolution de la flore microbienne}

Le compostage est un processus aérobie de dégradation des composés organiques par l'action successive des micro-organismes (bactéries et champignons) dont la concentration peut atteindre des millions par gramme de compost (Barje et al., 2008, Jouraiphy, 2007, Amir, 2005 ; Amir et al., 2010) ; leur évolution est en fonction des stades de compostage avec un profil défini, en fonction des espèces, à la nature et la structure des substrats et aussi liée principalement aux variations des paramètres physico-chimiques (Rosenzweig et al., 1980). Au cours du processus de compostage réalisé, la flore microbienne varie considérablement (fig.9). Par ailleurs, la densité bactérienne est toujours plus élevée que la densité fongique quel que soit l'âge du compost. 


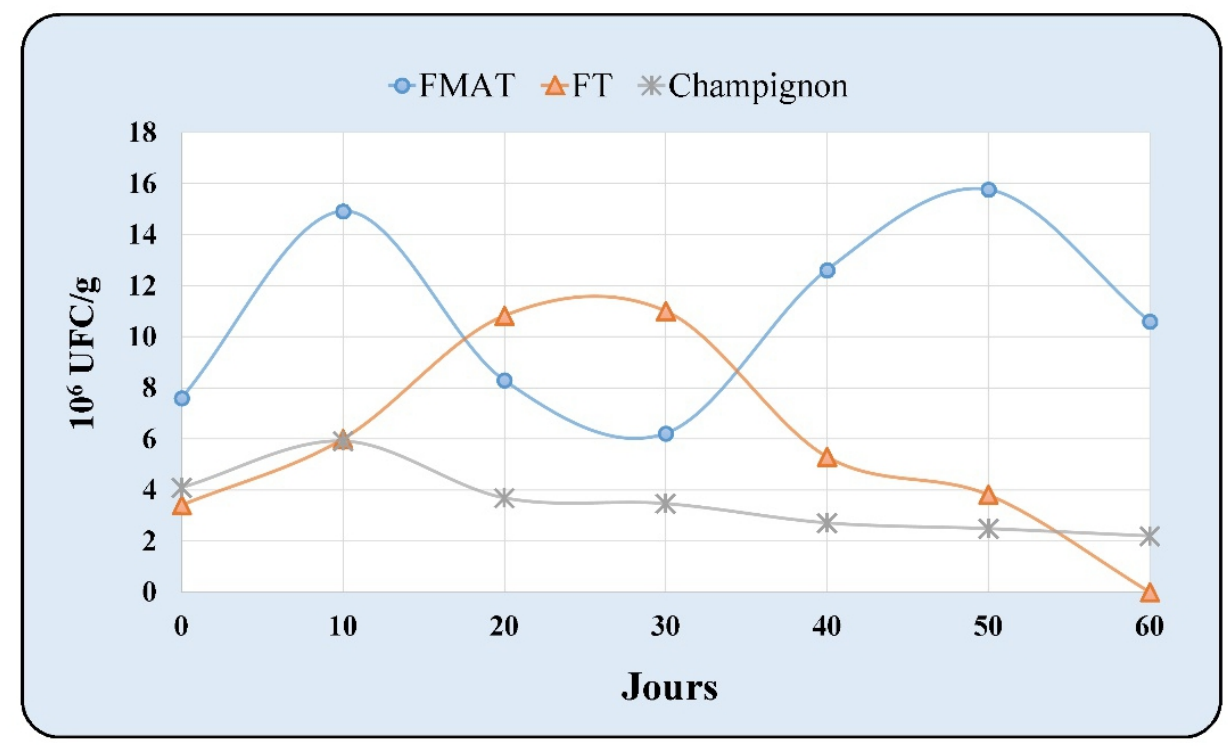

Figure 9 : Evolution de la flore microbienne durant la période du compostage.

Le début de la phase mésophile est caractérisé par une dominance de la flore mésophile qui est aux alentours de $3.10^{6} \mathrm{ufc} / \mathrm{g}$ et une colonisation du milieu par les coliformes totaux $10^{4} \mathrm{ufc} / \mathrm{g}$ et par les champignons $370 \mathrm{ufc} / \mathrm{g}$. Selon Kilbertus (1985), ce sont les champignons qui colonisent en premier les substrats et dégradent préférentiellement la matière organique fraîche mais l'augmentation de la température entraîne leur diminution et leur nombre passe donc de 370 à 40 ufc/g à partir du 20e jour jusqu'au 25e jour ceci est en accord avec les travaux de Greenberg et al., 1986 ; Guene, 2002 qui ont déduit que la baisse du nombre de champignon est en raison d'installation de conditions peu favorables à leur prolifération. Vers le 20e jour, la phase thermophile s'installe, la température est de l'ordre de $64,3{ }^{\circ} \mathrm{C}$, on assiste à un abattement de 2 unités logarithmiques pour les coliformes fécaux le nombre des mésophiles diminue nettement. Ces derniers sont partiellement inactivés ou tués, et en contre-partie, le nombre des thermophiles augmente sensiblement et atteint alors $510^{4} \mathrm{ufc} / \mathrm{g}$ de vers le 25e jour.

Dans ce contexte, Beffa et al. (1996) trouvent que le genre Thermus est parmi le groupe de bactéries thermophiles qui domine dans la dégradation de la matière organique. Au cours de la phase de refroidissement après le $40^{\mathrm{e}}$ jour, il y a une réduction importante du nombre des thermophiles, ce qui plaide en faveur du bon déroulement du processus de compostage puisque leur nombre atteint $210^{2} \mathrm{ufc} / \mathrm{g}$. A la phase de maturation, on note un repeuplement du compost par la flore mésophile puisqu'elle atteint $310^{5}$ ufc/g. Ces micro-organismes sont les plus attendus pour deux raisons principales : ils accélèrent le processus de décomposition et donnent un 
compost mûre en éliminant les pathogènes transportés par divers composés de déchets. Ces microorganismes continuent de dégrader les substrats comme la cellulose, la lignine, etc., pour obtenir en fin de cycle un compost stable (Tuomela et al 2000 ; Veeken et al 2001 ; Bolta et al., 2003).

\section{Tests de phytotoxicité}

\section{Test de germination}

Le test de germination est un moyen d'évaluation de la toxicité liée à l'incorporation des composts immatures dans le sol de se rendre compte de la maturité d'un compost. Les résultats des essais de germination sur le compost pur ou mélangé au sol sont reportés dans le tableau 2.

Tableau 2: Test de germination de deux variétés de blés et de tomate sur compost pur ou mélangé au sol.

\begin{tabular}{|c|c|c|c|c|}
\cline { 2 - 5 } \multicolumn{1}{c|}{} & Sable (témoin) & $\begin{array}{c}\text { Sable+1/3 de } \\
\text { compost }\end{array}$ & $\begin{array}{c}\text { Sable + 2/3 de } \\
\text { compost }\end{array}$ & $\begin{array}{c}\text { Compost } \\
\mathbf{( 1 0 0 \% )}\end{array}$ \\
\hline Blé & 70 & 85 & 57 & 23 \\
\hline Tomate & 58 & 67 & 0 & 0 \\
\hline
\end{tabular}

Ces résultats montrent que l'incorporation d'une dose de $25 \%$ du compost au sol permet un taux de germination de $85 \%$ pour le blé et 67 $\%$ pour la tomate. En revanche, les cultures sur un substrat contenant $75 \%$ à $100 \%$ du compost sont plutôt réprimées. Ces résultats sont en accord avec ceux rapportés par De hann et Abad Berjon qui ont signalé que l'effet dépressif du compost n'est pas lié uniquement aux caractéristiques des composts, mais il est aussi en fonction des doses appliquées ( De Hann, 1981 ; Abad Berjon et al., 1997). La germination varie donc avec la dose du compost apporté et le type de culture (Compaoré et al.,2010).

\section{Indice de germination}

Les IG les plus significatifs sont obtenus avec les doses de $25 \%$ et $50 \%$ d'extrait aqueux des différents composts (Tableau 3).

Tableau 3 : Taux d'indices de germination (\%) des graines laitue sur les extraits de composts purs ou mélangés à l'eau distillée.

\begin{tabular}{|c|c|c|c|c|c|}
\hline Doses & $100 \% \mathrm{E}$ & $75 \% \mathrm{E}+25 \% \mathrm{EC}$ & $50 \% \mathrm{E}+50 \% \mathrm{EC}$ & $25 \% \mathrm{E}+75 \% \mathrm{EC}$ & $100 \% \mathrm{EC}$ \\
\hline Laitue & 100 & 77 & 67 & 61 & 46 \\
\hline
\end{tabular}

E: Eau distillée; $\quad$ EC: Extrait de Compost

D’après Zucconi et al., (1981), un compost est considéré comme non toxique lors que son IG dépasse 50\%. Une dose de $75 \%$ d'extrait de compost donne des indices de germination de $77 \%$ avec les graines de laitue. L'extrait de compost pur ( $100 \%$ EC) a donné des pourcentages d'indice de germination entre $46 \%$ pour la laitue. Ces résultats montrent que le taux 
d'indice de germination varie avec les doses d'extraits de compost et avec le type de la culture.

\section{Conclusion \& perspectives}

Dans le procédé de compostage aérobie, les micro-organismes ont besoin d'oxygène pour décomposer la matière organique. Dans cette étude, la consommation d'oxygène a été fourni par injection d'air en semi-continu à l'intérieur du bioréacteur. Les résultats obtenus montrent que les déchets ménagers peuvent être composté avec succès dans un délai de 60 jours. Les indices opérationnels tels que la température, $\mathrm{pH}$ et les gaz dégagés ont été très utiles pour évaluer la performance de compostage et révèlent une activité microbienne vigoureuse. Le compost produit dans cette étude a été satisfaisant pour son application agricole en termes de rapport $\mathrm{C} / \mathrm{N}$ et de la conductivité électrique comme un indice de sa teneur en sels, en plus il ne présente pas de problems de phyto-toxicité et contient des éléments nutritifs qui peuvent lui permettre de jouer un rôle de fertilisant.

\section{References:}

1. Abad Berjon M., M.D. Climent Morato, P.Aragon Revuelta. and A.Camarero Simon (1997). The influence of solid Urban Waste compost and nitrogen -mineral fertilizer on grow the and productivity in potatoes. Commun. Soil Sci . Plant Anal., 28:1653-1661.

2. Ahn H.K., Sauer T.J., Richard T.L., Glanville T.D. (2009) : Determination of thermal properties of composting bulking materials. Bioresource Technology, 100 : 3974-3981.

3. Amir S. (2005) : Contribution à la Valorisation de Boues de Stations d'Epuration par Compostage : Devenir des Micropolluants Métalliques et Organiques et Bilan Humique du Compost. Thèse de Doctorat, Institut National Polytechnique, Toulouse, France.

4. Amir S., Abouelwafa R., Medich A., Souabi S., Winterton P., Merlina G., Revel JC., Pinelli E., Hafidi M. (2010) : Plfas of the microbial communities in composting mixtures of agroindustry sludge with different proportions of household waste. International Biodeterioration and Biodegradation, 64 : 614-621.

5. Barje F., Amir S., Winterton P., Pinelli E., Merlina G., Cegarra J., Revel JC., Hafidi M. (2008) : Phospholipid fatty acid analysis to monitor the co-composting process of olive oil mill wastes and organic household refuse. Journal of Hazardous Materials, 154 : 682687.

6. Basalo, C. (1974). Les ordures ménagères en agriculture. T.S.M. L'Eau, 69 : 15-23. 
7. Beffa, T., Blanc, M., Lyon, P.F., Vogt, G., Marchiani, M., Fischer, J.L. et Aragno, M. (1996) : Isolation of thermus strains from hot compost $\left(60-80^{\circ} \mathrm{C}\right)$. Appl. Environ. Microbiol., 62 : 1723-1727.

8. Bolta S.V., Mihelic R., Lobnik F., Lestan D. (2003). Microbial Community Structure during Composting With and Without Mass Inocula. Compost Sci. Util. $11: 6-15$.

9. Chang, Y. (1967): The fungi of wheat straw compost. ii : Biochemical and physiological studies. Trans British Mycol Soc, 50 : 667-677.

10. Chang, Y. et Hudson, HJ (1967) : The fungi of wheat straw compost. i : Ecological studies. Trans British Mycol Soc, 50 : 649-666.

11. Chen, J. (1999). Characteristic and applications of domestic animal wastes. In Animal Waste Products Quality and Treatment Alternatives Manual, Soil Survey and Testing Center (pp. 15-22). National Chung Hsing University, Taiwan.

12. De Bertoldi, M., Vallini, G. and Pera, A. (1983). The biology of composting: a review Waste Management \& Research, 1:157-176.

13. De Hann, S. (1981). Results of municipal waste compost rescarch over more than fifty years at the Institute for Soil Fertility at Haren/ Gorningen, the Netherlands. Neth .J. agric.Sci, 29:49-61.

14. Djerrari M.F. (1993) : Élimination et valorisation des ordures ménagères par compostage, cas de l'U.T.O.M. de Rabat. Séminaire régional, l'environnement urbain, des approches municipales innovatrices, Marrakech-25-27 mai.

15. Dutruc-Rosset G. (2003). Techniques analytiques et de contrôle (Codex œnologique). Partie microbiologique. Office International de la Vigne et du Vin. 23 p.

16. El M'ssari A. (1993): Elaboration d'une méthodologie de caractérisation des ordures ménagères et du compost de l'usine de Salé. Thèse 3ème cycle. Faculté des Sciences Moulay Ismail, Meknès.

17. Golueke, C. G. (1977). The biological approach to solid Waste management. Compost Sci., 18 :, 4-9.

18. Golueke, C. G. (1979). Composting : a review of rationale, principles and public health. Compost Sci., $17: 11-15$.

19. Gómez-brandon, M., Lazcano, C. et Domínguez, J. (2008) : The evaluation of stability and maturity during the composting of cattle manure. Chemosphere, 70 : 436-444.

20. Gray, K.R., Sherman, K. et Diddlestone, A.J. (1971) : Un examen de compostage. Partie 1. Processus Biochem., 31 : 32-36.

21. Greenberg A.E., Shastid T.B., Ellgas W.M. (1986). Quality Controlling Monitoring. Biocycle 7 : 36-38. 
22. Guene O. (2002). Integrated Traditional Composting within Domestic Solid Waste Management. Proceedings of International Symposium on Environmental Pollution Control and Waste Management,Tunisia, 349-356.

23. Hassen A. et Belguith K. (2001) : Microbial characterization during composting of municipal solid waste. Bioresource Technology 80(3) : 217-225.

24. He X.T., Logan T.J.,Traina S.J. (1992). Physical and Chemical Characteristics of Selected U.S Municipal Solid Waste Composts. J. Environ. Qual. 24 : 543-552.

25. Hellmann B., Zelles L., Palojarvi A., Bai Q. (1997). Emission of Climate-Relevant Trace Gases and Succession of Microbial Communities during Open-Windrow Composting.Appl. Environ. Microbiol. 63 : 1011-1018.

26. Hirai, M., Chanyasak, V. et Kubota, H. (1983) : A standard measurement for compost maturity. Biocycl, 24 : 54-56.

27. Jemali B., Soudi B., Lhadi E. K. (1996) : Contrôle des paramètres du compostage et appréciation de la qualité du compost des déchets ménagers de la Wilaya de Rabat-Salé. Revue Marocaine des Sciences Agronomiques et Vétérinaires, 16 (2).

28. Jouraiphy A. (2007) : Compostage des boues activées déchets verts, analyse physicochimiques, microbiologiques, toxicologiques, bilan humique et valorisation agronomique, Thèse de doctorat, Université Caddi Ayyad, Faculté des sciences Semlalia, Marrakech.

29. Juste C. (1980) Avantages et inconvénients de l'utilisations des composts d'ordures ménagères comme amendement organique des sols ou supports. INRA Bordereaux. Journées internationales sur le compost. Madrid

30. Kilbertus G. (1985). Compostage des écorces. Rapport Université de Nancy I, 356p.

31. Kochtizky, O.W., Seaman, W.K. et Wiley, J. S. (1969) : Municipal Compostage research at Johnson City, Tennessee. Compost Sci., 9 : 5-16.

32. Lin, C. (2008) : A negative-pressure aeration system for composting food wastes. Biores Technol, 99 : 7651-7656.

33. Lu, Y. (2000): Analytical Methods on Soil Agrochemistry (in Chinese). China Agriculture Technology Press.

34. Morel, J. L., Guckert, A., Nicolardot, B., Benistant, D., Catroux, G. and Germon, .J.C. (1986). Etude de l'évolution des caractéristiques physico -chimiques et de la stabilité biologique des ordures ménagères au cours du compostage. Agronomie, 6 : 693-701. 
35. Mustin M. (1987) : Le compost, Gestion de la matière organique. Ed. François DUBUSC, Paris, 954 p.

36. Peters, S., Schwieger, S.K.F. et Tebbe, C.C. (2000) : Succession of microbial communities during hot composting as detected by pcrsingle-stran-conformation polymorphism-based genetic profiles of small-subunit rrna genes. Appl Environ Microbiol, 66 : 930-936.

37. Rapilly F. (1968). Les techniques de mycologie en pathologie végétale. Annales des Epiphytes. INRA. 19 : 102 p.

38. Rosenzweig et Statozky., 1980 : bacteria often possess a competitive advantage.

39. Ryckeboer J., Mergaert J., Vaes K., Klammer S., De Clercq D., Coosemans J., Insam H. et

40. Swings J. (2003) : A survey of bacteria and fungi occurring during composting and self-heating processes. Ann. Microbiol., 53 (4) : 349410.

41. Soudi B. \& Jemali B. (1995) : Problématique du compostage des déchets ménagers au Maroc : cas de Rabat-Salé, programme de la communauté européenne. Séminaire MED-URBS, Kénitra 26-27 juin.

42. Soumaré, M., Demeyer, A. et Tack, F. (2002) : Chemical characteristics of malian and belgian solid waste composts. Biores Technol, 81 : 97-101.

43. Strom, P.F. (1985) : Identification of thermophilic bacteria in solid waste composting. Appl Environ Microbiol, 50 : 906-913.

44. SWEEP NET Le réseau régional d'échange d'informations et d'expertises dans le secteur des déchets solides dans les pays du Maghreb et du Machreq : Rapport sur la gestion des déchets solides au Maroc, 2014.

45. Tiejen, C. (1975). The potential of composting in developing countries. Compost Sci.,16:6-7.

46. Tuomela, M., Vikman, M., Hatakka, A. et Itavaara, M. (2000) : Biodegradation of lignin in a compost environment : A review. Biores Technol, 72 : 169-183.

47. Veeken, A.H.M., Adani, F., Nierop, K.G.J., de Jagar, P.A. et Hamelers, H.V.M. (2001): Degradation of biomacromolecules during high-rate composting of whest straw-amended feces. J Environ Qual, 30 : 1675-1684.

48. Wei, F.S. (2002) : Analytic Method of Water Quality on Water and Wastewater (4th edn). China Environmental Science Press, Beijing. 18 : 4-9. 
49. Wong, J.W.C., Li, S.W.Y. et Wong, M. H. (1995) : Coal fly ash as a composting material for sewage sludge : effects on microbial activities. Environ Technol, $16: 527-537$.

50. Zucconi, F. et de Bertoldi, M. (1987) : Compost specifications for the production and characterization of compost from municipal solid waste. 